\title{
The Effect of Inter-bundle Resistive Barriers on Coupling Loss, Current Distribution and DC Performance in ITER Conductors
}

\author{
A. Nijhuis, Y. Ilyin, and H. H. J. ten Kate
}

\begin{abstract}
The role of inter-bundle resistive barriers (metal sheet wraps), introduced to reduce the inter-bundle coupling loss in multistage cabled Cable-In-Conduit Conductors (CICC) for the International Thermonuclear Experimental Reactor (ITER) is evaluated, based on results gained recently on short sample experiments in the Twente Cable Press and SULTAN. The obvious benefit of limiting the inter bundle coupling loss unavoidably goes together with impeding the redistribution of nonuniform currents in the coil winding introduced at the terminations, as well as reduction of the heat exchange between the bundles. Six-element numerical electromagnetic code simulations are presented that qualitatively explain the effect of wraps on the DC performance, strongly depending on the testing geometry. The computations illustrate that wraps can reduce the DC performance in short sample tests. At the same time simulations of the Poloidal Field Coil Insert (PFCI), with a winding length of $50 \mathrm{~m}$, have shown that omitting sub-stage wraps, can even degrade the DC performance of coils due to the short current transfer length in combination with current nonuniformity causing peak voltages in the most overloaded petals.
\end{abstract}

Index Terms-CICC, coupling loss, critical current, current distribution, interstrand resistance.

\section{INTRODUCTION}

T THE NbTi CICC's for ITER consist of more than thousand strands, $0.7 \mathrm{~mm}$ in diameter, cabled with transposition in several stages. The round cable with an internal cooling channel is enclosed in a conduit made of stainless steel [1]. The large size of the multi-strand cable goes along with the generation of coupling loss in the NbTi [2]-[4] and $\mathrm{Nb}_{3}$ Sn CICC's [5], [6]. The low frequency tests with sinusoidal applied field, as standard applied in the Cable Press [7] $(f \leq 0.2 \mathrm{~Hz})$, provide the representative coupling loss time constants for the loss in typical ITER coil field dump-rate scenario (exponential and linear) [8]. The results of the Cable Press imply that the last minus one cabling stage wrapping is mandatory to limit the coupling loss time constant to the ITER $n \tau$ design value of $150 \mathrm{~ms}$ for $\mathrm{Nb}_{3} \mathrm{Sn}$ and $100 \mathrm{~ms}$ for NbTi conductors. We mention here that the pure interstrand coupling loss in the PFIS CICC is four times higher for a conductor without wraps (NW) than with wraps (W), with an $n \tau$ of $240 \mathrm{~ms}$ after multi-cycling [4]. For a $\mathrm{Nb}_{3} \mathrm{Sn}$ TFMC conductor without wraps and a void fraction (VF) of $33 \%$, an $n \tau$ exceeding $400 \mathrm{~ms}$ may even be anticipated. The difference in

Manuscript received September 20, 2004. This work was supported in part by the EU under Contract EFDA-04/1136.

The authors are with the University of Twente, Faculty of Science and Technology, 7500 AE Enschede, The Netherlands (e-mail: a.nijhuis@tnw.utwente. nl).

Digital Object Identifier 10.1109/TASC.2005.869671 inter-stage contact resistance due to the wrapping also changes the current transfer length or in other words, the ability to redistribute the current between the last stage cable elements. In general, DC current nonuniformities are introduced at the joints where the current is inserted and leads to a lower performance in DC transport properties. In order to investigate the role of the wraps in the present conductor design, we compare tested conductors with similar layout, except for the metal sheet wraps covering the last minus one cabling stage. Results are available from the so-called Condopt/Elres Sub-Size (SS) conductors [9]-[11] and the PFIS full-size conductors [3] ,[4], [12], [13]. All NbTi conductors were tested in the Twente Cable Press and in SULTAN.

The DC performance with current nonuniformity introduced at the joints [3], is simulated numerically with the CUDI-CICC code [14] for the SULTAN short sample test geometry. The state of the art, common status of perception is that the application of sub-stage wraps is unfavorable for the DC performance [10], [15]. Here we show that this is not unconditionally valid for all cases and in particular for the Poloidal Field Coil Insert (PFCI) geometry [16].

A recognized potential drawback of the presence of wraps, not treated within the scope of this analysis, are the thermal gradients between central channel and cable region because the exchange of heat is hindered which may lead to local higher temperature, affecting the performance. This has been counterbalanced by reducing the wrapping coverage from $80 \%$ to $50 \%$ for improved helium flow.

\section{REsults ON CONDOPT/ElRES Sub-Size CONDUCTORS}

Two SS conductors with $288 \mathrm{Ni}$-plated strands of $0.70 \mathrm{~mm}$ strand- and $16.5 \mathrm{~mm}$ cable-diameter and a VF of $35 \%$ are similar except for the metal sheet wrapping around the four final sub-cables. The hairpin sample layout with position of terminations, voltage taps and high field region as tested in SULTAN is schematically shown in Fig. 1 [9], [10]. The distance from termination to the high field section where the actual voltage-current $(V I)$ measurement is done, amounts to $1.9 \mathrm{~m}$. At both terminations the Ni coating and sub-cable wraps were removed completely, the cable ends were swaged into a copper sleeve to a 20\% VF and filled with SnAg solder. Sections of both conductor types were tested in the Cable Press with cyclic mechanical transverse loading. The interstrand coupling loss is a factor of 2.3 higher for the conductor without wraps [11] and the ratio of the transverse inter-bundle contact resistances for $\mathrm{W}$ and $\mathrm{NW}$ 


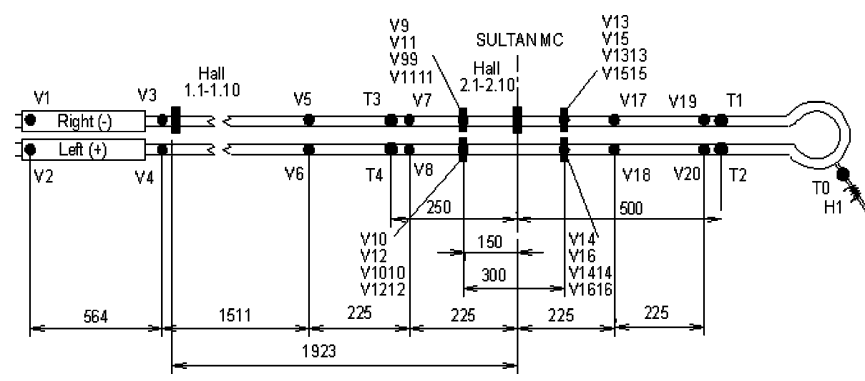

Fig. 1. Hairpin layout of the Condopt / Elres conductors in SULTAN.

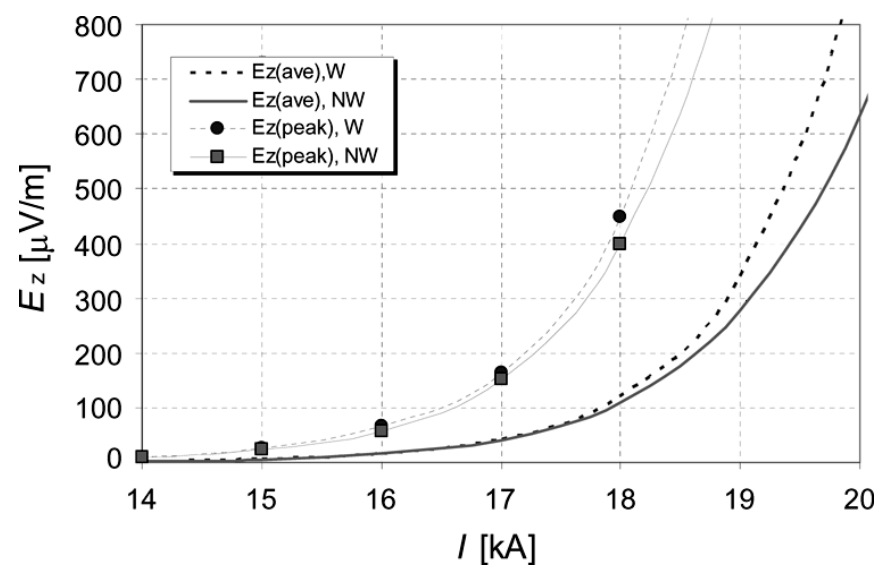

Fig. 2. The computed "measured" and "local peak" electric field in the high field section versus the transport current of both SS conductors in SULTAN, $B=5 \mathrm{~T}$ and $T=6.4 \mathrm{~K}$.

after 100 cycles is a factor 26 . An experimental $V I$-run is simulated with CUDI-CICC taking a similar geometrical layout and applied magnet field distribution with a maximum of $5 \mathrm{~T}$. For the measured inter-bundle resistance the values measured after 100 cycles in the press are adopted. The conductor self-field and the field from the parallel leg is taken into account. The temperature is $6.4 \mathrm{~K}$. The $R_{\mathrm{a}}$ (defined in [14] and clarified in [3]) is $2 \mu \Omega$ for NW and $50 \mu \Omega$ for $\mathrm{W}$. The $R_{\mathrm{a}}$ in the soldered termination is estimated at $0.2 \mu \Omega$, based on measurements at CRPP [17]. The joint (or termination) nonuniformity is based on a distribution defined in [14] as no data were available from this test. The level of nonuniformity is adopted as a more or less natural level of nonuniformity in the generally applied joint design, as deduced from self-field measurements by Hall sensor heads in other experiments [3]. The strand properties are taken from [9]. Redistribution of the current is allowed in both the regular cable and joint sections.

The results of the computation from the $V I$ curves are plotted in Fig. 2. Both lower curves correspond to the "average electric field" $\left(E_{z}\right)$ in the high field section, representing the $E_{\mathrm{z}}$ as it is measured in the experiment between the attached voltage taps. The actual $E_{z}$ profile along the four cable bundles varies a priori periodically due to the cable self field and depends secondly on the imposed current distribution among the bundles, causing transverse voltages between the strands and bundles [3]. This causes local peaks $E_{\mathrm{z} \text { (peak) }}$ in the longitudinal voltage distribution $E_{z}$ that are also plotted in Fig. 2. The local voltages in the cable can be much higher (in this case four times at $I_{\mathrm{c}}=18 \mathrm{kA}$ )

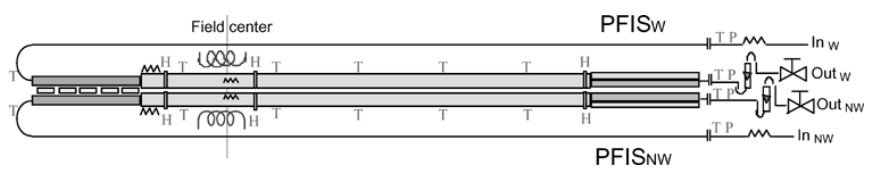

Fig. 3. Twin sample layout of the PFIS in SULTAN.

than what is generally measured between voltage taps attached to the conduit. We also find that the influence of the wraps on the $V I$ relation is relatively small for this configuration. This is due to the low transverse resistance in the termination in combination with the relatively short (compared to coils) distance of $1.9 \mathrm{~m}$ between the termination and the section in high magnet field allowing only modest current redistribution in the cable section. In [10] it was reported that in the SULTAN experiment $\mathrm{W}$ is more sensitive to current unbalance in the joint, leading to about $1 \mathrm{kA}$ reduction in $I_{\mathrm{c}}$ (at $100 \mu \mathrm{V} / \mathrm{m}$ ) for the same field and temperature conditions. The quench current is quite near the critical current and is slightly higher for NW, which is qualitatively in agreement with the simulation.

\section{RESULTS PFIS CONDUCTORS}

The two PFIS conductors with $1440 \mathrm{Ni}$-plated strands of $0.73 \mathrm{~mm}$, cable diameter of $37 \mathrm{~mm}$, central cooling channel and a VF of $34 \%$ are practically similar except for the metal sheet wrapping around the six final sub-cables (petals) [12]. The test configuration with the short distance of $0.1 \mathrm{~m}$ between the joint and the high field section in SULTAN is depicted in Fig. 3. A key difference between both conductors is in the joint itself, where the sub-cable wraps for $\mathrm{W}$ are only removed at the cable outer surface and not between the petal contact interfaces. So the inter bundle resistance in the joint section is higher for conductor $\mathrm{W}$ in the PFIS.

For the CUDI-CICC computation the temperature is fixed at $6.6 \mathrm{~K}$ and the applied field at $5 \mathrm{~T}$. The $R_{\mathrm{a}}$ outside the joint region is based on the Cable Press measurements [11] and amounts to $3 \mu \Omega$ for NW and $60 \mu \Omega$ for $\mathrm{W}$. The $R_{\mathrm{a}}$ in the joint is $0.1 \mu \Omega$ for NW and $1 \mu \Omega$ for $\mathrm{W}$ (due to the pieces of the wrapping left in-between the strand bundles of PFIS-W). The effect of a lower and higher inter-bundle contact resistance are computed for $0.1 \mu \Omega$ and $5 \mu \Omega$ for $\mathrm{W}$ [17] to evaluate the effect of a joint similar to NW and also the influence of a higher transverse resistance on the conductor performance, as unexpectedly observed in the PFIS test [12]. The joint (and termination) nonuniformity is based on the distribution deduced from Hall probe self-field analysis and transverse voltage measurements [3], [14]. The strand properties are taken from [12].

The computed results are presented in Fig. 4. The bold lower curves correspond to the average $E_{z}$ (representing the "measured" $E_{z}$ ) and show only a modest difference between NW and $\mathrm{W}$. The observed difference is primarily due to the difference in the joint transverse resistance and to less extent from the cable transverse resistance. Taking similar transverse resistance in the joint section for $\mathrm{W}$ and NW $\left(R_{\mathrm{a}}=0.1 \mu \Omega\right)$ gives no relevant difference in the computed $V I$-curves (for both $E_{\mathrm{z}(\text { ave })}$ and $E_{\mathrm{z}(\text { peak) }}$ ) of both legs (not shown in Fig. 4). This is because the (computed) current redistribution process, with increasing transport current, occurs primarily in the joint section and barely 


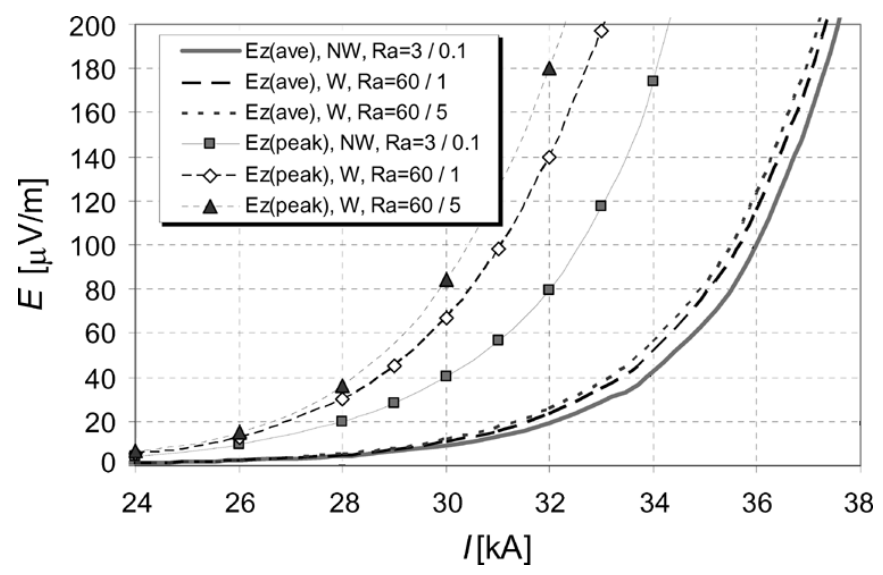

Fig. 4. The computed "measured" and local peak electric field in the high field section versus the transport current.

along the cable. Even if we increase the transverse resistance in the joint section from PFIS-W by a factor of 5 , hardly any increase of the average ("measurable") voltage turns up.

However, the voltage correlated to the peak field $E_{\mathrm{z} \text { (peak) }}$ computation not only rises much earlier (several kA's) in W compared to NW, but shows a noticeable sensitivity for the joint transverse resistivity $(\sim 1 \mathrm{kA})$ and hardly for the resistivity in the cable section. This means that the behavior of both legs in the SULTAN geometry is dominated by the difference between the joint sections and not by the wraps in the cable section.

\section{COMPUTATION OF PFCI PERFORMANCE}

A description of the coil and the field profile on the conductor is given in [16]. The field profile on the PFCI conductor winding is rather flat. The distance from the high field region to the intermediate joint is less than $1 \mathrm{~m}$ and to the lower joint about $2 \mathrm{~m}$. For the CUDI-CICC computation we consider only the main winding of the PFCI. The $R_{\mathrm{a}}$ for the cable winding section is based on the PFIS measurements. The $R_{\mathrm{a}}$ in the joint is now taken as $0.5 \mu \Omega$ for both NW and W to separate the pure cable wrap influence from possible joint differences between $\mathrm{W}$ and NW. The distribution in the joint connective resistances is similar to that from the PFIS configuration. The $T=6.6 \mathrm{~K}$ and $B$ from CSMC is $4.6 \mathrm{~T}$, the PFCI self-field is included in the computation.

The computed VI curves are presented in Fig. 5. In the coil center there is no noticeable difference in the average $E_{\mathrm{z}}$ for W or NW (the curves coincide) but the computed peak voltages differ. The initial alteration observed for the $V I$-curve in the case with wraps is a typical current redistribution phenomenon. In contradiction to the SS and PFIS results presented above, the $E_{\mathrm{z} \text { (peak) }}$ is higher for the case without wraps in the PFCI. The longer current transfer length in the case with wraps restrains redistribution in the cable section and nearly all of the current must be redistributed in the joints. Fig. 6 illustrates that the current distribution in the case with wraps does not become uniform in the center of the winding. Without wraps the current distribution is practically uniform in the winding center but is allowed to become more nonuniform toward the joints due to the low transverse resistivity (short current transfer length). This causes significantly higher peak voltages in the overloaded petals for

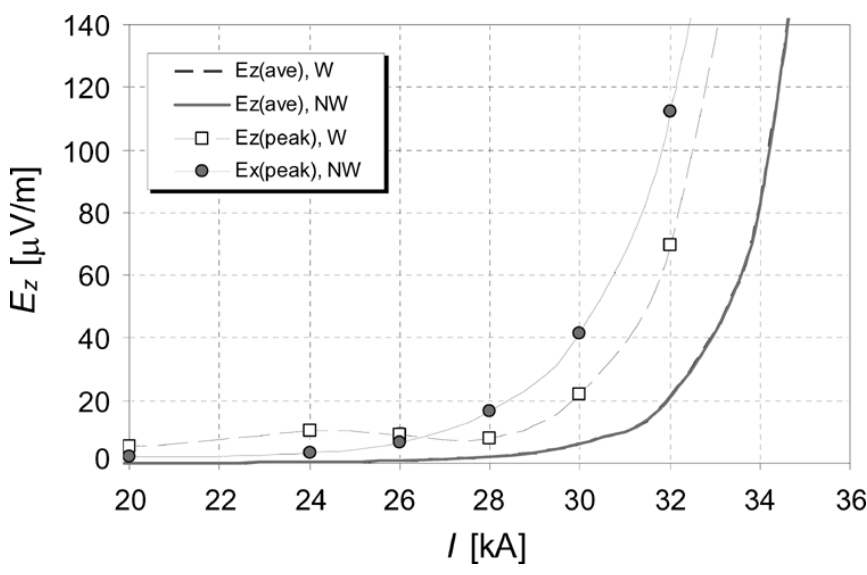

Fig. 5. The computed "measured" and local peak electric field in the central winding section versus the transport current with $\mathrm{Ra}$ values representing the values after 100 cycles of the PFIS $\mathrm{W}$ and NW samples, $T=6.6 \mathrm{~K}, B=$ $4.6 \mathrm{~T}$.

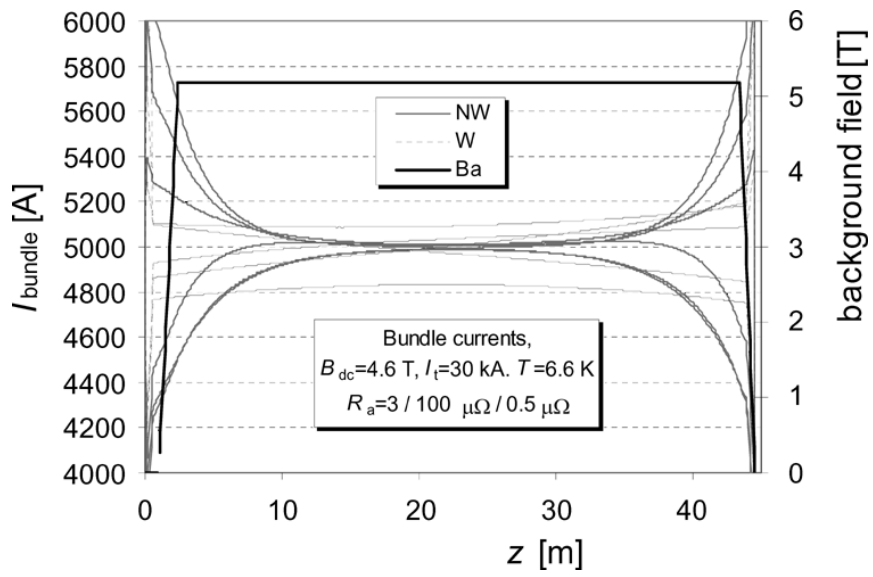

Fig. 6. The computed current distribution profile for the petals along the PFCI main winding for the case with and without wraps.

the case without wraps as illustrated in Fig. 7. The periodicity of peaks in the voltage along the length is caused by the supposition of cable self-field and applied field corresponding with the last-minus-one cabling stage pitch. If the current transfer length or evolution of the petal currents from joints to more uniformity is characterized by exponential decay, then the spatial decay constant is about a few meters for NW and one order of magnitude higher for $\mathrm{W}$ in the PFCI.

\section{DISCUSSION}

The experimental AC loss results show that sub-cable wraps are required on NbTi ITER conductors in order to limit the coupling loss. According to the model computations, in spite of the common opinion, it is not obvious that a higher inter-bundle resistance, impairing the redistribution of the bundle currents, at the same time degrades the DC performance in ITER coils. A shorter current transfer length from a lower transverse interpetal resistivity, allows a substantial dispersion in the redistribution between the petals along the winding thus increasing the peak voltages in the overloaded petal of the cable, eventually decreasing the DC performance. A drawback of the higher transverse cable resistivity due to the wraps is the somewhat higher 


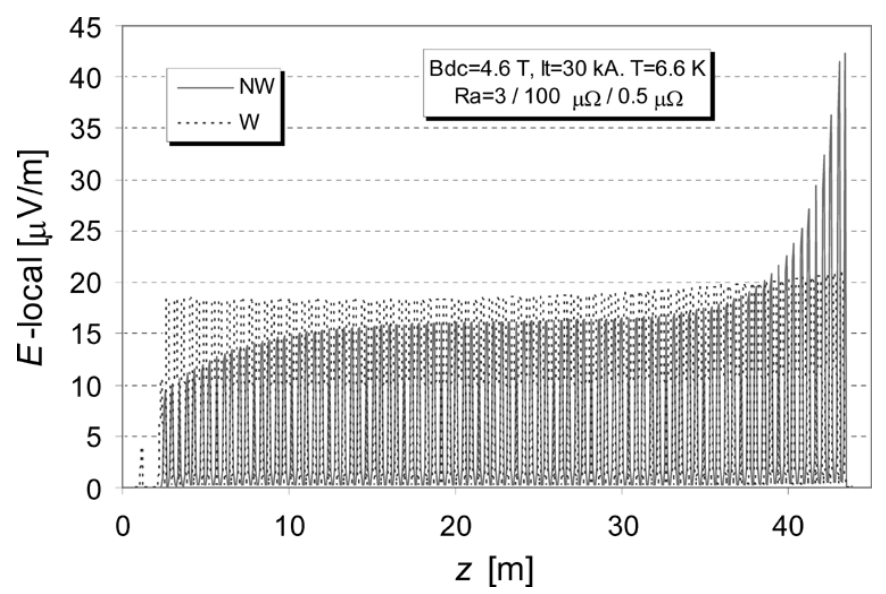

Fig. 7. The computed local electric field $\left(E_{z}\right)$ in the most overloaded cable bundles with highest peak voltages in the cases with and without wraps (W and NW).

joint redistribution loss, as most of the current redistribution is forced to take place in the joint and less in the cable winding section, although this depends also on the length of the winding.

For the SS conductors we find a slightly better performance for NW due to a modest redistribution in the cable section. From the PFIS full-size test we can not conclude straightforward on the role of the wraps at the cable section as the difference in DC performance is mainly attributed to the dissimilar joint layout. The average $E_{\mathrm{z}}$ does not show relevant differences but the main difference is in the local peak $E_{\mathrm{z}}$ highly depending on the joint resistivity. In that sense the computations are in agreement with the experimental results showing a better performance for the PFIS-NW and confirming that both conductors are below expectations as pointed out in [12] and [13]. It is anticipated that the heat developed in the regions with peak voltages expands to the surrounding bundle area, causing an accumulated and accelerated heating, thus resulting in a steep take-off in the measured $V I$ curve. According to the model interpretation, the effect is not only caused by the self-field of the conductor but is distended by the current nonuniformity.

Regarding the assumptions and simplification made, it is not rational to expect a precise quantitative prediction for this plain model. That's why we did not extend to even more detailed quench predictions connected to local heating due to the peak voltage. Nevertheless, in all cases we find a good qualitative indication that the peak voltage plays an important role in the voltage take-off process.

\section{CONCLUSION}

Local high peak voltages are generated in the cable bundle, exceeding the average "measured" voltage, due to the combination of cable self-field, spatial applied field and current nonuniformity caused by the joints. These peak voltages seem respon- sible for an earlier and steeper rise of the cable $V I$ curve or may even result in a sudden take-off.

The cable sub-stage wraps effectively decrease the coupling loss by about a factor of four but can reduce the DC performance at short sample testing due to the short length for redistribution. At the same time simulations of the Poloidal Field Coil Insert (PFCI), with a winding length of $50 \mathrm{~m}$, have shown that shortening the current transfer length by omitting sub-stage wraps, can even degrade the DC performance of coils. This is due to a shorter current transfer length causing an enhanced dispersion of the current nonuniformity and thus higher peak voltages in the overloaded petals.

\section{REFERENCES}

[1] D. Bessette et al., "Conductors of the ITER magnets," IEEE Trans. on Appl. Supercond., vol. 11, pp. 1550-1553, March 2001.

[2] A. Nijhuis and Y. A. Ilyin et al., "Change of interstrand contact resistance and coupling loss in various prototype ITER NbTi conductors with transverse loading in the Twente Cryogenic Cable Press up to 40000 cycles," Cryogenics, vol. 44, pp. 319-339, May 2004.

[3] Y. Ilyin, A. Nijhuis, and H. H. J. ten Kate, "Interpretation of conduit voltage measurements on the Poloidal Field Insert Sample using the CUDI-CICC numerical code," Cryogenics, 2006, In press.

[4] Y. Ilyin and A. Nijhuis et al., "Effect of cyclic loading and conductor layout on contact resistance of full-size ITER PFCI conductors," IEEE Trans. on Appl. Supercond, vol. 15, p. 1359, 2005.

[5] A. Nijhuis and Y. A. Ilyin et al., "Performance of an ITER CS1 Model Coil Conductor under transverse cyclic loading up to 40000 cycles," IEEE Trans. Appl. Supercond., vol. 14, pp. 1489-1494, 2004.

[6] A. Nijhuis, Y. Ilyin, W. Abbas, H. H. J. ten Kate, M. V. Ricci, and A. della Corte, "Impact of void fraction on mechanical properties and evolution of coupling loss in ITER Nb3Sn conductors under cyclic loading," IEEE Trans. Appl. Supercond., vol. 15, pp. 1633-1636, 2004.

[7] W. Abbas and A. Nijhuis et al., "A fully automatic press for mechanical and electrical testing of full size ITER conductors under transverse cyclic load," Adv. in Cryog. Eng., Materials, vol. 50, pp. 51-58, 2004.

[8] N. Martovetsky, "Losses in the CSMC, preliminary observations" July 7, 2000, Memo.

[9] R. Wesche, A. Anghel, B. Stepanov, and P. Bruzzone, "DC performance of subsize NbTi cable-in-conduit conductors," IEEE Trans. on Appl. Supercond, vol. 14, pp. 1499-1502, 2004.

[10] B. Stepanov, A. Anghel, P. Bruzzone, and M. Vogel, "Impact of impressed current unbalance on n-index," IEEE Trans. on Appl. Supercond, vol. 14, pp. 1495-1498, 2004.

[11] Y. Ilyin and A. Nijhuis et al., "Electromagnetic performance of subsize NbTi CICC's subjected to transverse cyclic loading," IEEE Trans. Appl. Supercond., vol. 14, pp. 1503-1506, 2004.

[12] P. Bruzzone et al., "Test results of the ITER PF insert conductor short sample in SULTAN," IEEE Trans. on Appl. Supercond, vol. 15, pp. 1351-1354, 2005.

[13] D. Ciazynski et al., "DC Performances of ITER NbTi conductors: models vs. measurements," IEEE Trans. on Appl. Supercond, vol. 15, pp. 1355-1358, 2005.

[14] A. Nijhuis, H. G. Knoopers, Y. Ilyin, A. Godeke, B. ten Haken, and H. H. J. ten Kate, "Effect of self-field and current nonuniformity on the voltage-temperature characteristic of the ITER central solenoid insert coil by numerical calculations," Cryogenics, vol. 42, pp. 469-483, 2002.

[15] P. Bruzzone, "Superconductors and joints, tests and trends for future development," Fus. Eng. and Design, vol. 56-57, pp. 125-134, 2001.

[16] R. Zanino et al., "Preparation of the ITER poloidal field conductor insert (PFCI) test," IEEE Trans. on Appl. Supercond, vol. 15, pp. $1347-1350,2005$.

[17] P. Bruzzone, July 2005, CRPP, Villigen, CH, private communication. 